\title{
Alcohol consumption and cardiovascular disease incidence in men with and without hypertension: the Suita study
}

Aya Higashiyama, Tomonori Okamura, Makoto Watanabe, Yoshihiro Kokubo, Ichiro Wakabayashi, Akira Okayama and Yoshihiro Miyamoto

Hypertension Research (2013) 36, 89; doi:10.1038/hr.2012.140

Correction to: Hypertension Research (2013) 36, 58-64; doi:10.1038/

hr.2012.133; published online 30 August 2012

After the publication of this article, the authors noticed an error in the Abstract. The number of the participants in this study should have read 2336, as stated in the main article.

The error has now been rectified, and the correct article appears in this issue. The html and online pdf versions have also been rectified. 